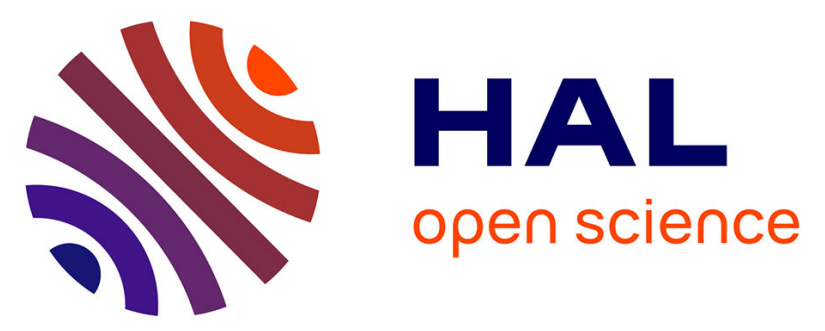

\title{
Combining noninvasive genetics and a new mammalian sex-linked marker provides new tools to investigate population size, structure and individual behaviour An application to bats
}

\author{
Diane Zarzoso-Lacoste, Pierre-Loup Jan, Lisa Lehnen, Thomas Girard, \\ Anne-Laure Besnard, Sebastien J. Puechmaille, Eric Petit
}

\section{To cite this version:}

Diane Zarzoso-Lacoste, Pierre-Loup Jan, Lisa Lehnen, Thomas Girard, Anne-Laure Besnard, et al.. Combining noninvasive genetics and a new mammalian sex-linked marker provides new tools to investigate population size, structure and individual behaviour An application to bats. Molecular Ecology Resources, 2018, 18 (2), pp.217-228. 10.1111/1755-0998.12727 . hal-01769650

\section{HAL Id: hal-01769650}

\author{
https://hal-univ-rennes1.archives-ouvertes.fr/hal-01769650
}

Submitted on 8 Jun 2018

HAL is a multi-disciplinary open access archive for the deposit and dissemination of scientific research documents, whether they are published or not. The documents may come from teaching and research institutions in France or abroad, or from public or private research centers.
L'archive ouverte pluridisciplinaire HAL, est destinée au dépôt et à la diffusion de documents scientifiques de niveau recherche, publiés ou non, émanant des établissements d'enseignement et de recherche français ou étrangers, des laboratoires publics ou privés. 
DR. DIANE ZARZOSO-LACOSTE (Orcid ID : 0000-0003-2992-4453)

Article type : Resource Article

Combining noninvasive genetics and a new mammalian sex-linked marker provides new tools to investigate population size, structure and individual behaviour: an application to bats

D. Zarzoso-Lacoste ${ }^{1,2, *}$, P-L. Jan ${ }^{3, *}$, L. Lehnen ${ }^{4}$, T. Girard ${ }^{3}$, A-L. Besnard ${ }^{3}$, S. J. Puechmaille , E. J. Petit ${ }^{3}$

*Co-first author

${ }^{1}$ Université Rennes 1, UMR 6553 ECOBIO Campus de Beaulieu, 263 avenue du Général Leclerc, 35042 Rennes Cedex, France

${ }^{2}$ Laboratoire Ecologie, Systématique \& Evolution, UMR 8079 - Université Paris-Sud/CNRS/AgroParisTech, Université Paris-Saclay, 91405 Orsay Cedex, France

${ }^{3}$ UMR985 ESE, Agrocampus Ouest, INRA, 65 rue de St-Brieuc, 35042 Rennes, France

${ }^{4}$ Greifswald University, Zoology Institute, Soldmannstr. 14, D - 17487 Greifswald, Germany

Keywords: noninvasive genetics, molecular sexing, Chiroptera, parentage assignments, Rhinolophus hipposideros

\section{Corresponding author:}

Diane Zarzoso-Lacoste

Université Paris Sud

Laboratoire Écologie, Systématique et Évolution

Équipe Écologie des Populations et des Communautés

362 Rue du Doyen André Guinier

91405 ORSAY Cedex

lacoste_diane@yahoo.fr 
Running title: sex-linked behaviours and social structure

\section{Abstract:}

Monitoring wild populations is crucial for their effective management. Noninvasive genetic methods provide robust data from individual free-ranging animals, which can be used in capture-mark-recapture (CMR) models to estimate demographic parameters without capturing or disturbing them. However, sex- and status-specific behaviour, which may lead to differences in detection probabilities, is rarely considered in monitoring. Here, we investigated population size, sex ratio, sex- and status-related behaviour in 19 Rhinolophus hipposideros maternity colonies (Northern France) with a noninvasive genetic CMR approach (using faeces) combined with parentage assignments. The use of the DDX3X/YMam sexual marker designed in this study, which shows inter- and intra-chromosomal length polymorphism across placental mammals, together with 8 polymorphic microsatellite markers, produced high quality genetic data with limited genotyping errors and allowed us to reliably distinguish different categories of individuals (males, reproductive and nonreproductive females) and to estimate population sizes. We showed that visual counts represent well adult female numbers and that population composition in maternity colonies changes dynamically during the summer. Before parturition, colonies mainly harbour pregnant and non-pregnant females with a few visiting males whereas after parturition, colonies are mainly composed of mothers and their offspring with a few visiting non-mothers and males. Our approach gives deeper insight into sex- and status-specific behaviour, a prerequisite for understanding population dynamics and developing effective monitoring and management strategies. Provided sufficient samples can be obtained, this approach can be readily applied to a wide range of species. 


\section{Introduction}

Monitoring wild populations is crucial for their effective management (McMahon et al. 2011; Pereira et al. 2013). Yet, developing robust monitoring protocols is challenging (Nichols \& Williams 2006), particularly regarding detection probability issues (Vos et al. 2000; Yoccoz et al. 2001; Pollock et al. 2002). While extrinsic factors (related to observer identity, time, climate, habitat, or site) that lead to detection probability variation can be accounted for using covariates, this is not the case when variation results from inter-individual variation in behaviour. A common source of intra-specific variation is sex, with males and females displaying different behaviour that leads to large differences in detection probabilities (e.g. Ogutu et al. 2006; Christy et al. 2010; Singh et al. 2014). This observation lead to the development of statistical frameworks accounting for specific forms of intra-specific variation (e.g. Veech et al. 2016), but the most obvious and efficient way of correcting potential bias is to a priori identify subsets of populations that differ in detection probabilities.

Numerous bat species are threatened by human induced perturbations and global change, and their loss could dramatically impact ecosystem functions and services (Mickleburgh et al. 2002; Jones et al. 2009; Rebelo et al. 2010). Monitoring programs that are set up to assess bat population trends most often use visual counts or, more recently, passive acoustics. One important caveat of these approaches is that different categories of individuals, which are not identifiable during census, may differ in detection probabilities. For example, sexual segregation varies both spatially and temporally in bats and many other organisms (Wearmouth \& Sims 2008; Angell et al. 2013). Different behaviour may even occur between individuals of the same sex (Senior et al. 2005), potentially leading to differences in detection probabilities (Marescot et al. 2011). Moreover, visual counts may cause disturbance to roosting bats, especially during the parturition and hibernation periods 
(Kunz et al. 2009). Despite these limitations, visual counts are widely used in maternity colonies to study bat population structure and dynamics, either by assuming that males are not a substantial component of maternity colonies (Johnson et al. 2011; Olson \& Barclay 2013) or by acknowledging that the unknown amount of males within colonies does not permit to correctly estimate essential parameters such as fecundity (Seckerdieck et al. 2005). When unverified, the first assumption is particularly likely to lead to incorrect inferences on population structure and dynamics. Furthermore, because females are the most important contributors to population dynamics in bats and many other animals, it is crucial to be able to differentiate both sexes when estimating colony sizes.

Capture-mark-recapture (CMR) techniques deliver individual level data which allow insights into bat life history and ecology, including longevity, physiology, social organization, movement behaviour, reproduction, sex ratio, and, to some degree, survival and population size (O'Shea et al. 2004; Ellison 2008). However, CMR methods have generally proven unsuccessful for reliably estimating bat population size due to bias associated with the need to capture individuals multiple times, heterogeneity in individual detection probability, low recapture rates, trap happiness or shyness, or mark loss (Kunz 2003; Schorr et al. 2014). Therefore, CMR is difficult to apply to bats, which are elusive and sometimes rare organisms that are usually small-sized, nocturnal, cryptic, highly mobile and capture-sensitive. Superior censusing methods should minimize disturbance and sampling bias (Yoccoz et al. 2001; Kunz et al. 2009).

Noninvasive genetic methods have become a well-established tool to study free-ranging animals by identifying each individual through its unique DNA fingerprint, without having to capture or disturb them. DNA is extracted from noninvasive samples (hair, faeces, urine) and amplified at genetically informative markers. As a drawback, noninvasive samples often contain only low amounts of degraded target DNA, resulting in genotyping errors (Broquet $e t$ 
al. 2006). Previous work has proven that high quality genotypes can be efficiently obtained from bat feaces (Puechmaille et al. 2007). Multilocus genotypes are then treated as individual molecular marks, and identical genotypes are considered as recaptures. This information can be used in CMR models to estimate demographic parameters (Lukacs \& Burnham 2005). The data provided can also be analysed using population genetic tools to gather critical information on sex-ratio (using a sex-linked genetic marker), relatedness, pedigrees, population structure or genetic diversity within or between populations (Waits \& Paetkau 2005).

Rhinolophus hipposideros is the Palearctic bat species whose population decline is best documented. During the last century, it became rare or extinct at the northern edge of its range in Northern Europe, likely due to pesticides use, food shortage, and habitat loss (Bontadina et al. 2000; Weiner \& Zahn 2000; Farcy et al. 2009). Females are known to return to their natal roost in late spring or early summer to give birth and raise their single young (Gaisler 1966). Males are usually assumed to live alone or to gather in small groups throughout the year but their presence in maternity roost has already been reported (Gaisler \& Chytil 2002; Bontadina et al. 2002). Pregnant and lactating females have stricter roost requirements than others (Speakman et al. 2003). Such possible intra-specific variations in roosting requirements and behaviour depending on sex or reproductive status could result in intrinsic heterogeneity in detection probabilities, and therefore, affect colony size (i.e. total number of adults) and fecundity (i.e. ratio juveniles/adults) measurements when estimated by visual counts (Safi et al. 2007; Veech et al. 2016). Here, we provide evidence that genetic approaches, including molecular sexing, are of great interest to elucidate the population structure of bat maternity colonies and to investigate possible differences in sex- and/or status-related behaviours.

The main goals of this study were to investigate population size, sex ratio and sex- 
related behaviour in $R$. hipposideros maternity colonies with a noninvasive genetic CMR approach combined with parentage assignment. We developed a robust molecular protocol, using 8 polymorphic microsatellites and one sexual marker which we applied on samples from $19 R$. hipposideros colonies situated in Northern France, assessing the importance of taking into account sex and reproductive status when monitoring populations.

\section{Material and Methods}

\section{Study site and sampling protocol}

19 colonies located in Northern France (Picardie) were investigated (Fig 1). Bat faeces were collected during two successive sessions conducted before and after parturition in 2013. Newspaper was spread on the ground under the main hanging sites of each colony to gather bat faeces. After approximately 10 days, faeces were collected and stored on containing silica gel fragments to prevent DNA degradation until analysis (Puechmaille \& Petit 2007). The number of visible adults was recorded during each visit.

\section{DNA isolation}

All pre-PCR procedures were carried out with aerosol barrier tips and single-use non-talc gloves, and pre- and post-PCR procedures were performed in separate rooms to avoid crosscontamination. For each colony, the desired number of faeces (twice the number of bats visually counted during colony sampling; see Table 2) was randomly picked. Faeces were individually homogenized using two glass beads $(2 \mathrm{~mm}$ diameter) for $2 \times 30 \mathrm{~s}$ at $30 \mathrm{~Hz}$ (Tissuelyser, Retsch). DNA was extracted using the NucleoSpin® 96 Plant II Kit (MachereyNagel) with slight modifications from the original protocol (Appendix A).

\section{Development of a new polymorphic sex-linked marker}

A primer pair was designed to target a pseudo-autosomal portion of the $8^{\text {th }}$ intron of the sex- 
linked DEAD-box helicase 3 gene (DDX3). This gene was previously amplified in bats, with amplicon lengths suitable for degraded DNA analyses (Hellborg \& Ellegren 2003). The new primer pair (DDX3X/Y-Mam-F: CAGATCTATGAGGAAGCCAGAAA; DDX3X/Y-Mam-R: TCATACCGCTCTAGAGTTCGC) targets a locus that exhibits different length on the X (154 bp from the DDX3X gene, also called DBX) and Y (131 bp from the DDX3Y gene, also called DBY) chromosomes, respectively.

The suitability of this marker for $R$. hipposideros sex identification was tested using 22 male and 17 female pure DNA samples extracted from postmortem biopsies following a salting out protocol (Petit et al. 1999). PCR reactions were conducted in a final volume of $15 \mu \mathrm{L}$ containing $1 \mu \mathrm{L}$ of template DNA, 10X PCR Buffer (20 mM Tris-HCl ph 8.4, $50 \mathrm{mM}$ $\mathrm{KCl}$; Promega), 0.2mM dNTPs (Invitrogen), 1.5mM MgCl2 (Promega), $0.3 \mu \mathrm{M}$ of forward and reverse primer, $0.05 \mathrm{U} / \mu \mathrm{L}$ Taq DNA polymerase (Promega). The cycling conditions included an initial denaturing step of $95^{\circ} \mathrm{C}$ for $15 \mathrm{~min}$ followed by 45 cycles of $94^{\circ} \mathrm{C}$ for $45 \mathrm{~s}$, $56^{\circ} \mathrm{C}$ for $45 \mathrm{~s}$, and $72^{\circ} \mathrm{C}$ for $1 \mathrm{~min}$ and a final extension step of $72^{\circ} \mathrm{C}$ for $1 \mathrm{~h}$. The PCR products obtained from two female and two male were cloned and purified (Strataclone PCR Cloning Kit, Agilent). Consensus sequences for DDX3 fragments amplified from both X- and Y-chromosomes were obtained by forward/reverse sequencing seven clones from each individual (Sanger technology).

In silico PCR (ecoPCR programme: Ficetola et al. 2010) was conducted to assess the theoretical coverage $(\mathrm{Bc})$ of our sex-linked primer pair. To do so, we compiled a customized sequence database by blasting (Blastn) our consensus X-linked amplicon on the entire EMBL database, and by retrieving from GenBank the mRNA sequences of each available mammalian (sub)species (taxid: 40674). We then filtered out those that (i) did not align correctly, (ii) were too short to cover the entire length of the corresponding amplicon (i.e partial sequences) and (iii) contained ambiguous nucleotides (e.g. N). We randomly kept one 
sequence per (sub)species to reduce overrepresentation of a few species. Sequences were trimmed to contain only the amplicon (including primer binding sites), and internal insertions were removed. Our customized mRNA sequence database, containing 91 unique mammalian (sub)species (Appendix B), was used with ecotaxstat (Ficetola et al. 2010) to compute Bc (allowing 3 mismatches).

We also looked for length polymorphism of our sex-linked marker in Mammalian species. We first compiled a customized sequence database by blasting (Blastn) our consensus X-linked amplicon on all available mammalian reference genomic sequences (refseq_genomic) and by retrieving the sequences (and corresponding chromosome, gene and sex annotations) from each matching (sub)species. We then filtered out those that did not align correctly, as well as partial sequences, and trimmed the sequences to contain only the amplicon (including primer binding sites). Finally, we calculated the theoretically amplified amplicon length for all the 162 sequences, belonging to 85 unique mammalian (sub)species, contained in our customized genomic sequence database (Appendix B).

\section{Multilocus genotyping}

Bat DNA was amplified and genotyped using 9 polymorphic molecular markers including a panel of 8 microsatellites selected from the literature, and our new sex-linked maker (Table 1, Appendix C). These 9 markers were optimized into a single $8 \mu$ multiplexed reaction containing 3.5 $\mu$ l Multiplex PCR buffer mix (Qiagen), $0.0875 \mu \mathrm{M}$ of each primer (Table 1) and $2 \mu 1$ DNA templates.

A touchdown thermal cycle program was used to prevent non-specific amplification, which included an initial denaturation at $95^{\circ} \mathrm{C}$ for 15 min followed by 16 cycles each of denaturation at $94^{\circ} \mathrm{C}$ for $45 \mathrm{~s}, 58^{\circ} \mathrm{C}$ with $0.5^{\circ} \mathrm{C}$ decrease per cycle for $45 \mathrm{~s}$, and extension at $72^{\circ} \mathrm{C}$ for $1 \mathrm{~min}$, and completed with 20 additional cycles with an annealing temperature of $50^{\circ} \mathrm{C}$. A final extension at $72^{\circ} \mathrm{C}$ for 1 hour was included at the end of the cycles before 
holding at $4^{\circ} \mathrm{C}$. Amplifications were replicated three times to allow for genotyping error detection and to build robust consensus genotypes. Alleles were scored using GeneMapper v.5 from electrophoreses run on a 3730xl DNA Analyzer sequencer with GeneScanTM500LIZ size standard (Applied Biosystems).

\section{Bioinformatic pipeline and genotyping quality}

An automatic procedure that scores genotypes and determines consensus genotypes was developed to avoid problems associated with subjective/inconsistent scoring of alleles and human errors and minimize the time necessary for scoring alleles. This bioinformatic pipeline is based on a comparison between alleles obtained from different scoring parameters for each replicate, and a comparison between replicates. A first scoring mode, "stringent", only considered peaks high enough to avoid genotyping errors associated with small peaks, with a threshold depending on the fluorescent dye (200 for PET, 100 for others). A second scoring mode, "non-stringent", employed peak height thresholds of 20, 40, 50, and 60 for FAM, VIC, NED, and PET, respectively. This allowed the recovery of peaks that were not detected with the first thresholds. Thresholds for stringent and non-stringent analyses were chosen after careful observation of randomly selected samples to account for the different markers' propensities to generate higher peaks or smaller artefacts. Peaks detected with the nonstringent analysis were only scored if they were detected in at least two of the three PCR replicates. Automated scoring with GeneMapper tends to not consider peaks obviously high enough when another peak is drastically higher. To overcome this issue, in a third approach, we scored our data as Amplified Fragment Length Polymorphisms (AFLP) instead of microsatellites. This allowed the detection of all the peaks within defined bins independently of the height of other peaks, retaining only those that had already been detected in previous scorings in at least one other replicate. When more than two alleles per locus were scored by the software, only the two highest were kept. 
The three replicates obtained from each sample were then used to create a consensus genotype by applying the following rule: one allele of the consensus must appear at least twice over the three replicates. In the rare case of problematic consensus (three alleles seen twice), the smallest peak was discarded. To rule out that distinct genotypes actually corresponded to the same individual because of genotyping errors, we manually checked every consensus that differed from others by one or two loci (mismatch 1 and 2 - Puechmaille \& Petit 2007). If there was any evidence for distinct genotypes originating from the same individual (i.e. if a particular allele was present in at least one replicate of the genotype of the individual scored as homozygous for that locus) this allele was retrospectively validated, in order to create a common genotype and merge samples.

Samples with missing data on at least one locus were discarded for subsequent analyses, except the juvenile dataset for parentage assignment (see below). Allelic Drop Out (ADO) and False Allele (FA) rates, as well as the Quality Index (QI), were estimated by comparing consensus genotypes to PCR replicates (Broquet \& Petit 2004; Miquel et al. 2006). The expected heterozygosity of each locus was computed with unique genotypes (i.e. individuals) using the adegenet package (Jombart 2008) to evaluate the markers' polymorphism.

Deviations from Hardy-Weinberg proportions were quantified and tested with FIS and the corresponding permutation test using the software GENETIX (Belkhir et al. 1996). Only individuals encountered before parturition (adults) were used in these two tests.

\section{Parentage assignment}

To identify mothers, parentage was assigned using the software Colony 2 with a full-pedigree likelihood method (Jones \& Wang 2010). Males were designated as polygamous, and mothers as monogamous with only one possible offspring because samples were taken over a single reproductive event (Gaisler 1966). Individuals sampled before parturition were considered as 
potential parents while those only sampled after parturition were considered as potential juveniles. Only individuals successfully genotyped at 9 loci were considered, except potential juveniles for which the selection threshold was lowered to 7 loci since these individuals, only sampled after parturition, were less likely to represent a clean genotype due to the lack of comparison between sampling sessions. We ran analyses ten times to assess the robustness of the results. Only individuals assigned at least five times out of ten runs were considered as parents.

\section{Population size estimates}

Population sizes were calculated using a Bayesian estimator based on single session CMR data (Petit \& Valière 2006). This method, adapted to noninvasive genetic data, considers every population size between a minimum (the number of genotypes detected) and a maximum size (set to 10 times the visual count) and determines the most probable population size given the distribution of recaptures (i.e. the number of faeces corresponding to the same individual). This method assumes a homogenous detection probability and a closed population. The homogeneity of detection probability was tested with the method developed in Puechmaille \& Petit (2007), which consists in simulating the sampling process under an assumption of homogeneous capture probability, and to compare the observed with the expected number of captures per individual. Population closure is highly likely since samples were deposited over a maximum of two weeks. Population size estimates were computed when considering adult (i) females and males and (ii) females only. Individuals assigned as juveniles were removed from population size estimates. To compare visual and genetic estimation methods, we performed a generalized linear mixed model (glmm) with population size as a response variable (following a Poisson distribution) and with the estimation method and sampling session as fixed effects. Colonies were considered a random effect. Fixed effect significances were tested with a Wald chi square test followed by post-hoc pairwise least- 
square means test. Glmm and Chi-square tests were performed in R 3.2.2 (R Core Team 2016). For comparison, we also estimated the population size with the Capwire package (Pennell et al. 2013), by performing either the "Equal Capture Model" or the "Two-Innate Rates Model" (depending on the likelihood ratio test included in the package) before performing the same glmm and subsequent tests. To validate the suitability of our molecular markers to discriminate among a large number of individuals, population size estimates were compared with the reciprocal of the probabilities of identity computed for unrelated individuals (PID-rand) and full siblings (PID-sibs) using Gimlet (Valière 2002) and for samples collected before parturition.

\section{Sex related behaviour}

The overall and offspring sex-ratio of each colony (i.e. the ratio of males to total number of individuals genotyped) was estimated separately before and after parturition. To investigate the capture probability of females and males depending on the sampling session, we performed a generalized linear mixed model $(\mathrm{glmm})$ with the number of recaptures for each individual as a response variable (following a Poisson distribution) and with sex and sampling session as fixed effects. Because the same individual could be sampled before and after parturition, we considered individuals as a random effect. The fixed effect significance was tested with a Wald chi-square test followed by a pairwise least-square means post-hoc test. A Chi-square test was performed to investigate if the inter-session recapture rate (number of individuals captured before and after parturition) differed between sexes and with female reproductive status. 


\section{Results}

\section{Molecular protocol validation and sex ratio}

The results of the amplification tests carried out with the DDX3X/Y-Mam sex-linked marker on 37 known $R$. hipposideros males and females confirmed its reliability for sex identification (100\% were correctly sexed). Sequenced amplicons provided one allele from the X-Chromosome (154 bp) and two from the Y-Chromosome (129 and $131 \mathrm{bp})$. Furthermore, in silico PCRs confirmed the reliability of the DDX3X/Y-Mam marker for sex identification, its length polymorphism between and within 85 unique mammalian (sub)species, and the wide taxonomic coverage of the corresponding primer pair that theoretically amplified $88 \%$ of the 91 tested (sub)species belonging to all described placental mammal super-orders (detailed results are available in Appendix B).

In this study, 3544 samples out of $5099(69.5 \%)$ were genotyped at all 9 loci. Mean ADO and FA rates over loci were $6.7 \%$ and $2.5 \%$ respectively, resulting in a QI of $91.6 \%$. The average expected heterozygosity of the eight microsatellite loci was 70\% (range 43-83\%). Over all loci and colonies, the minimal number of individuals that could be discriminated for unrelated individuals (1/PID-rand) and assuming full siblings (1/PID-sibs) was above $1.46 \mathrm{x}$ $10^{6}$ and $2.77 \times 10^{4}$, respectively, and was always clearly greater than the corresponding colony sizes estimated visually or genetically (Table 2).

We distinguished 1337 unique genotypes, inferred to correspond to different individuals, $345(25.8 \%)$ of which were males. The number of unique genotypes identified for each colony and sampling session ranged from 4 to 200 with a mean of 43.7, and was in the range of corresponding visual counts (Table 2). $F_{I S}$ values were variable between colonies, with four significantly positive values (Pic4, Pic5, Pic6, Pic19) and one significantly negative (Pic8). Males were sampled in each colony and at each sampling session, except in the smallest colony (Pic1) before parturition. The mean overall sex-ratio of 
the sampled colonies was 0.22 (range $0-0.5$ ) and varied between colonies and sampling sessions (Table 2).

\section{Population size estimates}

Population sizes estimated from all individuals ranged from 8 to 235 , with a mean of 64.16 $(\mathrm{SD}=46.16)$ individuals before parturition, and $60.28(\mathrm{SD}=34.49)$ after parturition (Table 2). When only considering females, average population sizes decreased to $47.9(\mathrm{SD}=28.71)$ and 44.56 ( $\mathrm{SD}=24.49)$ before and after parturition, respectively. However, capture probabilities were not homogenous in half of the colonies (in 9 to 11 colonies depending on male presence and sampling session, see Table 2). Because no recapture occurred at Pic1 after parturition, we were unable to estimate its population size.

Mean population sizes calculated from all individuals, before and after parturition, were greater than those estimated from females only. Mean adult population sizes estimated through visual counts were closer to those calculated from females only (Fig. 2) and no significant difference was observed between them before parturition (Least-square means, pvalue $=0.22$ ). Independently of the estimation method, adult population sizes decreased after parturition. Capwire estimates gave similar results, with no significant differences between visual counts and female population size before and after parturition, with a p-value of 0.25 and 0.32 , respectively (data not shown).

\section{Sex ratio and sex related behaviour}

Among the 662 females sampled before parturition and the 670 potential juveniles, only 174 were assigned as mother-juvenile pairs at least 5 times among 10 Colony runs. Motherjuvenile assignments were possible for all 19 sampled colonies, but with very heterogeneous proportions of mothers, varying from $8 \%$ to $80 \%$ (31.4\% on average). This analysis allowed us to distinguish mothers and unassigned females (hereafter, non-mother females). 
The number of samples collected from individuals assigned as mothers was significantly higher than for males for both sampling sessions (Least-square means, p-values $<0.05)$. The number of recaptures for mothers was particularly high after parturition, and significantly higher than for non-mothers (Fig. 3). There were significantly more inter-session recaptures for mothers $(63.8 \%)$ than for non-mothers $(34.2 \%)$, and more for both types of females than for males $(20.6 \%)$ (Chi-square test; p-values < 0.001).

\section{Discussion}

\section{Molecular protocol validation}

We developed a widely applicable noninvasive molecular protocol, involving eight microsatellite loci and one sex-linked marker, for population genetic studies conducted on $R$. hipposideros. Our results confirmed the suitability of the DDX3 sex-linked marker for reliable molecular-sexing from DNA extracted from different sources, including noninvasive samples. Indeed, the amplification of different pseudo-autosomal DNA fragment sizes (i.e. length polymorphism) from both $\mathrm{X}$ and $\mathrm{Y}$ chromosomes in a single PCR reaction allowed the unequivocal distinction between sexes by avoiding misidentification due to PCR failure (i.e. false negative results) when using chromosome-specific markers. We showed that this interchromosomal length polymorphism, which is linked to an indel located in the $8^{\text {th }}$ intron of the DEAD-box helicase 3 gene, is observed in 85 mammalian (sub)species, making it a potentially widely applicable molecular sexing tool (Appendix B.2).

Despite the challenging low concentration and degraded nature of noninvasive DNA samples, our protocol allowed the complete genotyping of $70 \%$ of analysed faeces and provided high quality genetic data (QI 91.6\%) with low genotyping error rates (ADO <7\% and FA $<3 \%$ ). When including the samples with missing data, the Quality Index dropped to $73.3 \%$ but $\mathrm{ADO}$ and FA rates remained relatively stable (6.6\% and 3.2\%, respectively). These 
values are among the best recorded when compared to other studies directly amplifying nuclear DNA from faeces (Broquet et al. 2006), but are lower than those obtained in Puechmaille et al. (2007), where microsatellites markers were only genotyped if mtDNA was previously amplified in a given sample. This suggests that directly performing three PCR replicates per sample can represent a good compromise between sufficiently reducing genotyping error rates while keeping the cost of large scale studies as low as possible.

Here, 1337 unique genotypes were obtained from 19 colonies, which, in combination with the low PIDs calculated for our data set (even assuming full siblings), demonstrates that our molecular marker set is variable enough to discriminate a large number of individuals.

$\mathrm{F}_{\text {IS }}$ spanned a wide range of values around zero, with the most extreme ones being significantly different from zero (Table 2). No particular locus was involved in these extreme values (data not shown), excluding potential experimental artefacts. These $\mathrm{F}_{\mathrm{IS}}$ values may rather be related to different underlying structures (e.g. Wahlund effect or family structures, see Parreira \& Chikhi 2015) in the different colonies, a topic that will require further investigation.

\section{Extrinsic factors affecting detection probability and population size estimates}

Mean bat colony sizes estimated visually were always closer to the genetic estimates of female population sizes than to those of the total population (Fig. 2). Hence, visual counts, which do not allow sex differentiation, underestimate the total number of individuals that occupy colonies. Indeed, individuals which are not present when the visual count occurs are missed. Individuals with the highest philopatry, i.e. females, are more likely to be detected. Beyond sex, individual detection probability may be heterogeneous due to a multitude of time- or site-dependent extrinsic factors affecting the observation process itself (observers' skills, timing and duration of censusing) and/or conditions (environmental factors restraining visibility or observers' proximity to the animals and/or factors related to species biology and 
ecology) (Kunz et al. 2009; Veech et al. 2016). Our results confirm that visual counts represent a reliable and cost effective method for censusing the more settled individuals within colonies (i.e. adult females, see below) when only interested in bat demographic trends. However, when more detailed demographic data are required (e.g. population structure, reproduction or vital rates), sampling noninvasively over several consecutive days combined with CMR approaches represents a powerful alternative. Genotypes were obtained at a cost of $7.25 €$ per sample (consumables only) and approximately two months of lab work and data analysis were required to obtain clean consensus genotypes for all 19 colonies. This investment provided high quality data at the individual level and while minimizing both disturbance of colonies and the effects of extrinsic factors on individual detection probability and population size estimates (Petit \& Valière 2006; Puechmaille \& Petit 2007; Kunz et al. 2009).

Half of the population size estimates did not meet the assumption of homogeneous detection probability, and this heterogeneity can alter population size estimates (Link 2003). Therefore, population size estimates taken at the colony level should be treated carefully. Indeed, in most wild animal populations, standardizing sampling effort is helpful to reduce biases due to extrinsic factors, but will not totally eliminate heterogeneity, because other intrinsic variables, like inter-individual behavioural variations, will influence their detection probability through space and time (Yoccoz et al. 2001; Veech et al. 2016). It is thus crucial to define subsets of populations that differ in detection probability for a more accurate evaluation of key parameters such as population sizes or vital rates.

\section{Intrinsic factors affecting detection probability and demography}

Within a population, individuals may exhibit distinct types of behaviour that will make them more or less detectable. This heterogeneity in detection probability can be related to one or several intrinsic factors including age (e.g. Crespin et al. 2006), sex (e.g. Tavecchia et al. 2002), breeding or social status (e.g. Ogutu et al. 2006). 
Although intrinsic heterogeneity remains difficult to measure and to control for statistically (Veech et al. 2016), its underlying factors are important to identify and consider whenever possible, because behaviour is often the primary force responsible for changes in density, genetic diversity, and sex or age structure.

\section{Sex related behaviour}

The set of molecular markers used allowed us to assess variation of bat maternity colony structure over time. Our results show that these colonies are indeed mainly composed of females but also that the number of males is substantial $(25.8 \%)$. This is in agreement with sex-ratios recorded in other European maternity colonies (Bontadina et al. 2002), though our results demonstrate that sex-ratio is not homogenous between colonies and through time within the same colony.

Unsurprisingly, a greater number of samples was analysed per female than per male before and after parturition (Fig 3). This number significantly increased in females while it decreased in males after parturition, suggesting that most males leave or spend less time in the roost and are not recaptured (Table 3). This is supported by the fact that our female population size estimates were slightly lower ( $13.7 \%$ on average) than visual counts, which only encompass the most settled individuals independently of their sex. Such behaviour has been observed in many other temperate bats where females segregate from males in spring and summer to form maternity colonies and raise their young (McCracken \& Wilkinson 2000; Senior et al. 2005; Safi et al. 2007; Ibáñez et al. 2009). Indeed, during this period of low mating willingness, the lower fidelity of males to maternity colonies may reduce local resource competition with pregnant and lactating females that display more stringent foraging and roosting requirements (Senior et al. 2005; Safi et al. 2007).

These results highlight the potential of molecular approaches to investigate sex-related 
behaviour, in relation to species biology and ecology, and to evaluate how it could affect individual detection probabilities during population surveys. Such sex-specific information can inform conservation programs, especially in social and/or colonial species in which females, which are key individuals for population growth, form isolated groups to give birth and raise their young, a period in which they are particularly vulnerable.

\section{Female reproductive status}

The combination of our noninvasive CMR approach with parentage analysis allowed deeper insights into the structure of maternity colonies, revealing that female roost fidelity can change over time according to reproductive status, and providing fecundity estimates.

Only $26.3 \%$ of the females sampled before parturition were assigned a mother status. This is surprisingly low considering previously reported values of this species' fecundity (e.g. 0.38 and 0.5 in Schofield 1996 and Petit et al. 2014, respectively). A possible explanation would be that we failed to assign a significant number of mothers due to insufficient sampling, or because of genotyping failure of some mothers before parturition and/or some juveniles after. The latter is particularly likely because sex-ratios of assigned juveniles, which are supposed to be balanced (Gaisler 1966), were biased towards females in our study (21.8\% males, data not shown). This could be explained by an unexpected difficulty to sample juveniles (e.g. particular composition and/or frequency of deposition of juvenile faeces, coprophagy behaviour of the mother), or by a significant part of the assigned juveniles actually corresponding to philopatric adult or subadult females sampled only after parturition. Nevertheless, variation in offspring sex-ratio has been reported in numerous mammal species including bats (Barclay 2012), and different adaptive explanations have been proposed (Clutton-Brock \& Iason 1986). 
We cannot currently accept or reject one of those two hypothetical explanations for the low number of assigned mothers observed, the reality being probably a mix of both. If low juvenile sampling prevails in shaping the observed patterns, sampling twice in a single year might not be optimal for correctly identifying all mothers of that year, or to estimate fecundity. Sampling colonies twice a year over multiple years would enhance both adult and offspring sample sizes and could help elucidating the causes of the observed low parentage assignment and female-biased offspring sex ratios.

Differences between the fecundity observed in bibliography, estimated from visual counts, and our molecular approach could also be related to intrinsic heterogeneity in individual detection probabilities due to behavioural differences in roost occupancy between individuals of different reproductive status. Indeed, the mean number of samples analysed per individual after parturition was higher for females assigned as mothers than for any nonmother in both sampling sessions (Fig. 3). For mothers, the number of samples analysed per individual increased after parturition, suggesting a smaller mobility of females that gave birth. This number did not vary for non-mother females between sampling sessions, suggesting a stable behaviour over this period. Furthermore, the proportion of females resampled after parturition was higher for females assigned as mothers (Tab. 3). These results clearly show that all females seem to frequent the maternity colony before the permanent settlement of mothers. Because the costs and benefits of site fidelity for females may change with reproductive conditions and associated energetic requirements, we hypothesize that nonmothers are less faithful to their colony because of intra-specific competition for local resources with mothers (see Anthony et al. 1981; Kunz et al. 2009; Ngamprasertwong et al. 2014).

Finally, our results highlight the suitability of our approach (involving a well conserved polymorphic pseudo-autosomal sexual marker) to (i) gather high quality data at the 
individual level, (ii) decipher complex inter-individual behavioural variations that may influence individual detection probability, and (iii) consider these factors for more precise population size and reproduction rate estimates, which are fundamental for efficient management of wild populations.

\section{Conclusion}

With noninvasive genetic sampling and an analysis method adapted to large datasets, we obtained high quality genetic data with minimal disturbance of the colonies. We showed that distinguishing males from females, using a sex-linked marker, offers unique opportunities to more reliably determine different categories of individuals (males, females, potential breeders, reproductive individuals), to identify extrinsic and intrinsic factors of heterogeneity in individual detection probabilities, and to estimate population sizes, which is critical for understanding population dynamics and optimise wildlife management. By combining parentage with CMR analyses, we showed that the population structure of $R$. hipposideros in maternity colonies changes dynamically during the summer. Parentage assignment provided valuable insights into female behaviour revealing that, after parturition, maternity colonies are mainly composed of mothers and their offspring while males and non-mother females continue to frequent them without residing there permanently. Because reproductive females crucially contribute to the establishment and long-term persistence of social animal colonies, disentangling behaviour specific for these different categories of individuals is essential for an effective monitoring and conservation of wild populations. Finally, our study highlights that noninvasive genetic samples, when analysed both as individual marks (CMR) and genetic fingerprints (parentage) instead of as allelic frequencies only, allow to investigate both individual (behaviour, mating, etc.) and population (genetics and dynamics) level processes. When combined with information on other biotic or abiotic factors, such data offer unprecedented information to better understand population dynamics and the underlying responsible 
processes (Jan et al. 2017). This knowledge is urgently needed for conservationists, managers and policy makers to understand how species are responding to global environmental change (e.g. climate, habitat) and to make long-term predictions.

\section{Acknowledgements:}

The National Forest Office, PROCOPE/DAAD and Research Training Group - DFG RTG 2010 provided funding. J. Lefèvre, P. Janin, J. Jaminon, V. Berger and B. Marette from the National Office Forest, as well as M. Georges, D. Top, A. Pierroux and T. Cheyrezy from the Conservatory of natural areas provided field and logistical support. E. Meglecz and V. Dubut provided support in taxonomic coverage analysis. E. Hartmann and A. Monchaux provided informatics support. S. Dool and E. Teeling from University College Dublin provided us with an unpublished $R$. hipposideros DBY8 sequence used for primer design.

\section{References:}

Angell RL, Butlin RK, Altringham JD (2013) Sexual segregation and flexible mating patterns in temperate bats. PLOS one, $\mathbf{8}$, e54194.

Anthony ELP, Stack MH, Kunz TH (1981) Night roosting and the nocturnal time budget of the little brown bat, Myotis lucifugus: effects of reproductive status, prey density, and environmental conditions. Oecologia, 51, 151-156.

Barclay RM (2012) Variable variation: annual and seasonal changes in offspring sex ratio in a bat. PLOS one, 7, e36344.

Belkhir K, Borsa P, Chikhi L, Raufaste N, Bonhomme F (1996) GENETIX 4.05, logiciel sous Windows TM pour la génétique des populations. Laboratoire Génome, Populations, Interactions, CNRS UMR 5000, Université de Montpellier II, Montpellier (France).

Bontadina F, Arlettaz R, Fankhauser T et al. (2000) The lesser horseshoe bat Rhinolophus hipposideros in Switzerland: present status and research recommendations. Le Rhinolophe, 14, 69-83.

Bontadina F, Schofield H, Naef-Daenzer B (2002) Radio-tracking reveals that lesser horseshoe bats (Rhinolophus hipposideros) forage in woodland. Journal of Zoology, 258, 281-290.

Broquet T, Ménard N, Petit E (2006) Noninvasive population genetics: a review of sample source, diet, fragment length and microsatellite motif effects on amplification success and genotyping error rates. Conservation Genetics, 8, 249-260.

Broquet T, Petit E (2004) Quantifying genotyping errors in noninvasive population genetics. Molecular Ecology, 13, 3601-3608.

Christy MT, Yackel Adams AA, Rodda GH, Savidge JA, Tyrrell CL (2010) Modelling detection probabilities to evaluate management and control tools for an invasive species. Journal of Applied Ecology, 47, 106-113.

Clutton-Brock TH, Iason GR (1986) Sex ratio variation in mammals. The Quarterly Review of Biology, 61, 339-374.

Crespin L, Harris MP, Lebreton J-D, Wanless S (2006) Increased adult mortality and reduced breeding success with age in a population of common guillemot Uria aalge using marked birds of unknown age. Journal of Avian Biology, 37, 273-282. 
Dool SE, Puechmaille SJ, Dietz C et al. (2013) Phylogeography and postglacial recolonization of Europe by Rhinolophus hipposideros : evidence from multiple genetic markers. Molecular Ecology, 22, 4055-4070.

Ellison L (2008) Summary and analysis of the US Government bat banding program.

Farcy O, Le Rouxel A, Queau S (2009) Activité et terrains de chasse du Petit rhinolophe (Rhinolophus hipposideros Bechstein, 1800) en Bretagne, France. Arvicola, 19, 12-20.

Ficetola GF, Coissac E, Zundel S et al. (2010) An In silico approach for the evaluation of DNA barcodes. BMC Genomics, 11, 434.

Gaisler J (1966) Reproduction in the lesser horseshoe bat (Rhinolophus hipposideros hipposideros Bechstein, 1800). Bijdragen tot de Dierkunde, 36, 45-62.

Gaisler J, Chytil J (2002) Mark-recapture results and changes in bat abundance at the cave of Na Turoldu, Czech Republic. Folia Zooligica, 51, 1-10.

Hellborg L, Ellegren H (2003) Y chromosome conserved anchored tagged sequences (YCATS) for the analysis of mammalian male-specific DNA. Molecular Ecology, 12, 283-291.

Ibáñez C, Guillén A, Agirre-Mendi PT et al. (2009) Sexual segregation in Iberian noctule bats. Journal of Mammalogy, 90, 235-243.

Jan P-L, Farcy O, Boireau J et al. (2017) Which temporal resolution to consider when investigating the impact of climatic data on population dynamics? The case of the lesser horseshoe bat (Rhinolophus hipposideros). Oecologia, 184, 749-761.

Johnson JB, Edwards JW, Ford WM (2011) Nocturnal Activity Patterns of Northern Myotis (Myotis septentrionalis) during the Maternity Season in West Virginia (USA). Acta Chiropterologica, 13, 391-397.

Jombart $\mathrm{T}$ (2008) adegenet: a $\mathrm{R}$ package for the multivariate analysis of genetic markers. Bioinformatics, 24, 1403-1405.

Jones G, Jacobs DS, Kunz TH et al. (2009) Carpe noctem: the importance of bats as bioindicators. Endangered Species Research, 8, 93-115.

Jones OR, Wang J (2010) COLONY: a program for parentage and sibship inference from multilocus genotype data. Molecular Ecology Resources, 10, 551-555.

Kunz TH (2003) Censusing bats: challenges, solutions, and sampling biases. In: Monitoring trends in bat populations of the United States and territories: problems and prospects (eds O'Shea TJ, Bogan MA), pp. 9 - 19. United States Geological Survey, Information and Technology Report USGS/BRD/ITR-2003-003, Washington, District of Columbia.

Kunz TH, Betke M, Hristov NI, Vonhof MJ (2009) Methods for assessing colony size, population size, and relative abundance of bats. In : Ecological and behavioral methods for the study of bats (2nd ed, eds Kunz TH, Parsons S), pp. 133 - 157. Johns Hopkins University Press, Baltimore, Maryland.

Link WA (2003) Nonidentifiability of population size from capture-recapture data with heterogeneous detection probabilities. Biometrics, 59, 1123-1130.

Lukacs PM, Burnham KP (2005) Research notes: estimating population size from DNAbased closed capture-recapture data incorporating genotyping error. Journal of Wildlife Management, 69, 396-403.

Marescot L, Pradel R, Duchamp C et al. (2011) Capture-recapture population growth rate as a robust tool against detection heterogeneity for population management. Ecological Applications, 21, 2898-2907.

McCracken GF, Wilkinson GS (2000) Bat mating systems. In: Reproductive biology of bats (eds Crichton EG, Krutzsch PH), pp. 3-5. Academic Press, San Diego, California.

McMahon SM, Harrison SP, Armbruster WS et al. (2011) Improving assessment and modelling of climate change impacts on global terrestrial biodiversity. Trends in Ecology 
\& Evolution, 26, 249-259.

Mickleburgh SP, Hutson AM, Racey PA (2002) A review of the global conservation status of bats. Oryx, 36.

Miquel C, Bellemain E, Poillot C et al. (2006) Quality indexes to assess the reliability of genotypes in studies using noninvasive sampling and multiple-tube approach. Molecular Ecology Notes, 6, 985-988.

Ngamprasertwong T, Piertney SB, Mackie I, Racey PA (2014) Roosting habits of Daubenton's bat (Myotis daubentonii) during reproduction differs between adjacent river valleys. Acta Chiropterologica, 16, 337-347.

Nichols J, Williams B (2006) Monitoring for conservation. Trends in Ecology \& Evolution, 21, 668-673.

Ogutu JO, Piepho H-P, Dublin HT, Reid RS, Bhola N (2006) Application of mark-recapture methods to lions: satisfying assumptions by using covariates to explain heterogeneity. Journal of Zoology, 269, 161-174.

Olson CR, Barclay RMR (2013) Concurrent changes in group size and roost use by reproductive female little brown bats (Myotis lucifugus). Canadian Journal of Zoology, 91, 149-155.

O'Shea TJ, Ellison LE, Stanley TR (2004) Survival estimation in bats: historical overview, critical appraisal, and suggestions for new approaches. In: Sampling rare or elusive species: concepts, designs, and techniques for estimating population parameters (ed Thompson W), pp. 297-336. Island Press, Washington, District of Columbia.

Parreira BR, Chikhi L (2015) On some genetic consequences of social structure, mating systems, dispersal, and sampling. Proceedings of the National Academy of Sciences, 112, E3318-E3326.

Pennell MW, Stansbury CR, Waits LP, Miller CR (2013) Capwire: a R package for estimating population census size from non-invasive genetic sampling. Molecular Ecology Resources, 13, 154-157.

Pereira HM, Ferrier S, Walters M et al. (2013) Essential Biodiversity Variables. Science, 339, 277-278.

Petit E, Excoffier L, Mayer F (1999) No Evidence of Bottleneck in the Postglacial Recolonization of Europe by the Noctule Bat (Nyctalus noctula). Evolution, 53, 12471258.

Petit E, Le Texier E, Farcy O (2014) Suivi démographique de quatre espèces patrimoniales en Bretagne : analyse statistique de 11 années de comptage. Symbioses, 32, 63-67.

Petit E, Valière N (2006) Contributed papers: Estimating population size with noninvasive capture-mark-recapture data: noninvasive capture-mark-recapture data. Conservation Biology, 20, 1062-1073.

Pollock KH, Nichols JD, Simons TR et al. (2002) Large scale wildlife monitoring studies: statistical methods for design and analysis. Environmetrics, 13, 105-119.

Puechmaille S, Mathy G, Petit E (2005) Characterization of 14 polymorphic microsatellite loci for the lesser horseshoe bat, Rhinolophus hipposideros (Rhinolophidae, Chiroptera). Molecular Ecology Notes, 5, 941-944.

Puechmaille SJ, Mathy G, Petit EJ (2007) Good DNA from bat droppings. Acta Chiropterologica, 9, 269-276.

Puechmaille SJ, Petit EJ (2007) Empirical evaluation of non-invasive capture-mark-recapture estimation of population size based on a single sampling session: Non-invasive capturemark-recapture. Journal of Applied Ecology, 44, 843-852.

R Core Team (2016) R: A Language and Environment for Statistical Computing. R Foundation for Statistical Computing. Vienna, Austria. https://www.R-project.org/.

Rebelo H, Tarroso P, Jones G (2010) Predicted impact of climate change on European bats in 
relation to their biogeographic patterns. Global Change Biology, 16, 561-576.

Rossiter SJ, Zubaid A, Mohd-Adnan A et al. (2012) Social organization and genetic structure: insights from codistributed bat populations. Molecular Ecology, 21, 647-661.

Safi K, König B, Kerth G (2007) Sex differences in population genetics, home range size and habitat use of the parti-colored bat (Vespertilio murinus, Linnaeus 1758) in Switzerland and their consequences for conservation. Biological Conservation, 137, 28-36.

Schofield HW (1996) The ecology and conservation biology of Rhinolophus hipposideros, the lesser horseshoe bat. Ph.D. Thesis. University of Aberdeen.

Schorr RA, Ellison LE, Lukacs PM (2014) Estimating sample size for landscape-scale markrecapture studies of North American migratory tree bats. Acta Chiropterologica, 16, 231239.

Seckerdieck A, Walther B, Halle S (2005) Alternative use of two different roost types by a maternity colony of the lesser horseshoe bat (Rhinolophus hipposideros). Mammalian Biology, 70, 201-209.

Senior P, Butlin RK, Altringham JD (2005) Sex and segregation in temperate bats. Proceedings of the Royal Society B: Biological Sciences, 272, 2467-2473.

Singh R, Qureshi Q, Sankar K, Krausman PR, Goyal SP (2014) Evaluating heterogeneity of sex-specific capture probability and precision in camera-trap population estimates of tigers. Wildlife Society Bulletin, 38, 791-796.

Speakman JR, Thomas DW, Kunz TH, Fenton MB (2003) Physiological ecology and energetics of bats. In: Bat ecology (eds Kunz TH, Fenton MB), pp. 430-490. The University of Chicago Press, Chicago, Illinois.

Struebig MJ, Kingston T, Petit EJ et al. (2011) Parallel declines in species and genetic diversity in tropical forest fragments. Ecology Letters, 14, 582-590.

Tavecchia G, Pradel R, Lebreton J-D, Biddau L, Mingozzi T (2002) Sex-biased survival and breeding dispersal probability in a patchy population of the Rock Sparrow Petronia petronia. Ibis, 144, E79-E87.

Valière N (2002) GIMLET: a computer program for analysing genetic individual identification data. Molecular Ecology Notes, 2, 377-379.

Veech JA, Ott JR, Troy JR (2016) Intrinsic heterogeneity in detection probability and its effect on N -mixture models. Methods in Ecology and Evolution, 7, 1019-1028.

Vos P, Meelis E, Ter Keurs WJ (2000) A framework for the design of ecological monitoring programs as a tool for environmental and nature management. Environmental monitoring and assessment, 61, 317-344.

Waits LP, Paetkau D (2005) Noninvasive genetic sampling tools for wildlife biologists: a review of applications and recommendations for accurate data collection. Journal of Wildlife Management, 69, 1419-1433.

Wearmouth VJ, Sims DW (2008) Sexual segregation in marine fish, reptiles, birds and mammals: behaviour patterns, mechanisms and conservation implications. Advances in Marine Biology, 54, 107-170.

Weiner P, Zahn A (2000) Roosting ecology, population development, emergence behaviour and diet of a colony of Rhinolophus hipposideros (Chiroptera: Rhinolophidae) in Bavaria. In: Proceedings of the VIIIth EBRS: Approaches to biogeography and ecology of bats (ed Woloszyn BW), pp. 231-242. Chiropterological Information Center, Institute of Systematics and Evolution of Animals PAS, Krakow, Poland.

Yoccoz NG, Nichols JD, Boulinier T (2001) Monitoring of biological diversity in space and time. Trends in Ecology \& Evolution, 16, 446-453.

\section{Data Accessibility:}

DNA sequences: Genbank accessions KY793696-KY793699 
$\mathrm{R}$ scripts and genotypes used in this study were deposited at DRYAD entry doi:10.5061/dryad.8fh54

\section{Author contributions:}

This project was designed by EJP with input from SJP and fundraising was conducted by EJP. Lab experiments were performed by DZL, PLJ, ALB and LL. Data were analysed by DZL, PLJ, TG, ALB, LL, SJP and EJP. The paper was written by DZL, PLJ, SJP and EJP. Both co-first authors contributed equally to the study. All authors were involved with data interpretation and draft corrections.

\section{Supporting Information:}

Appendix A: DNA isolation using the NucleoSpin® 96 Plant II Kit (Macherey-Nagel). Appendix B: In silico validation of our sex-linked primer pair.

Appendix C: Example of genotype profiles obtained from one male (A) and one female (B) (three replicates each). 


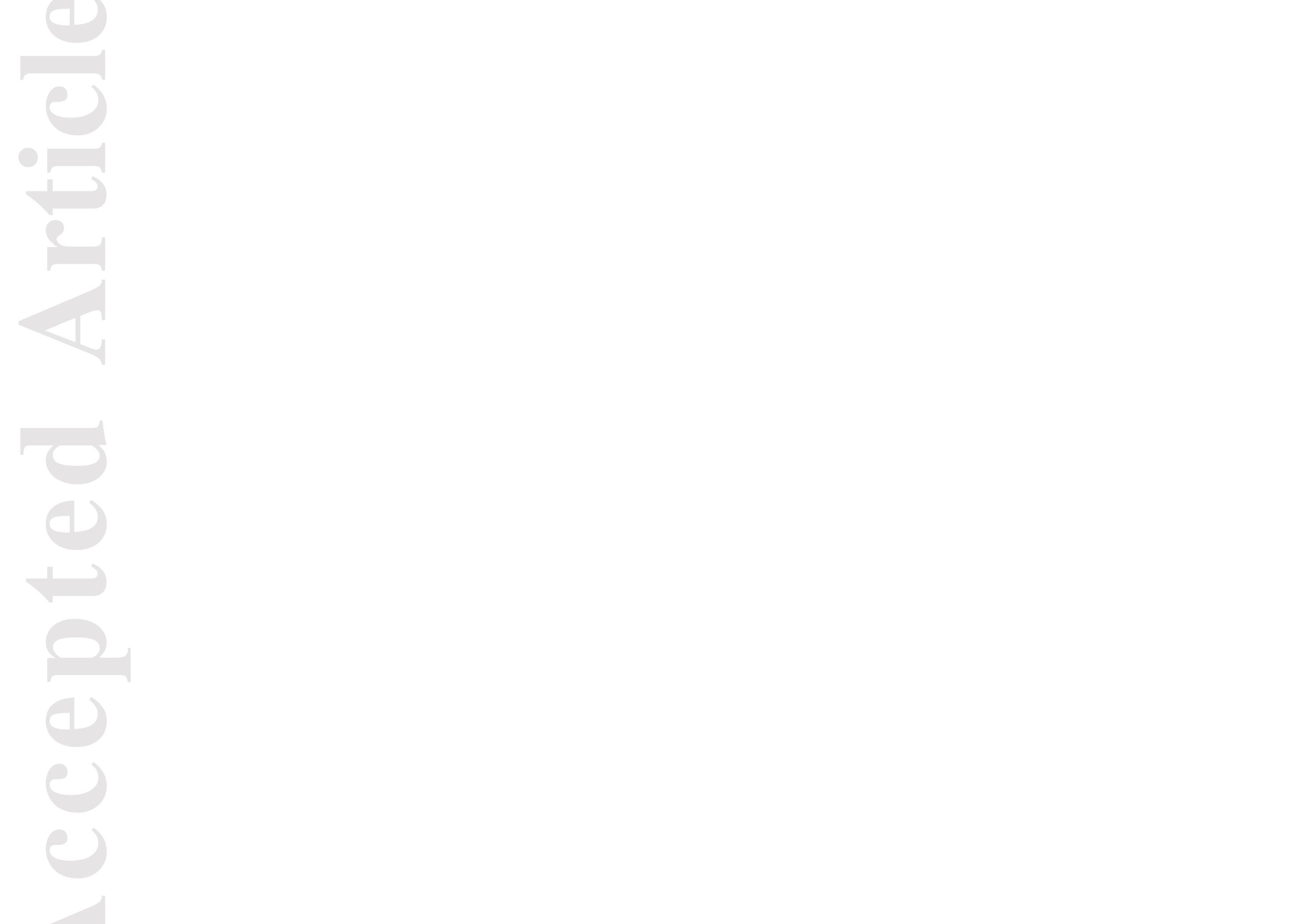


Table 1: Description, sources and amplification conditions of markers used this study and their respective measures of allelic drop-out rate (ADO), false allelic rate (FA), quality index (QI) and expected heterozygosity (Hs).

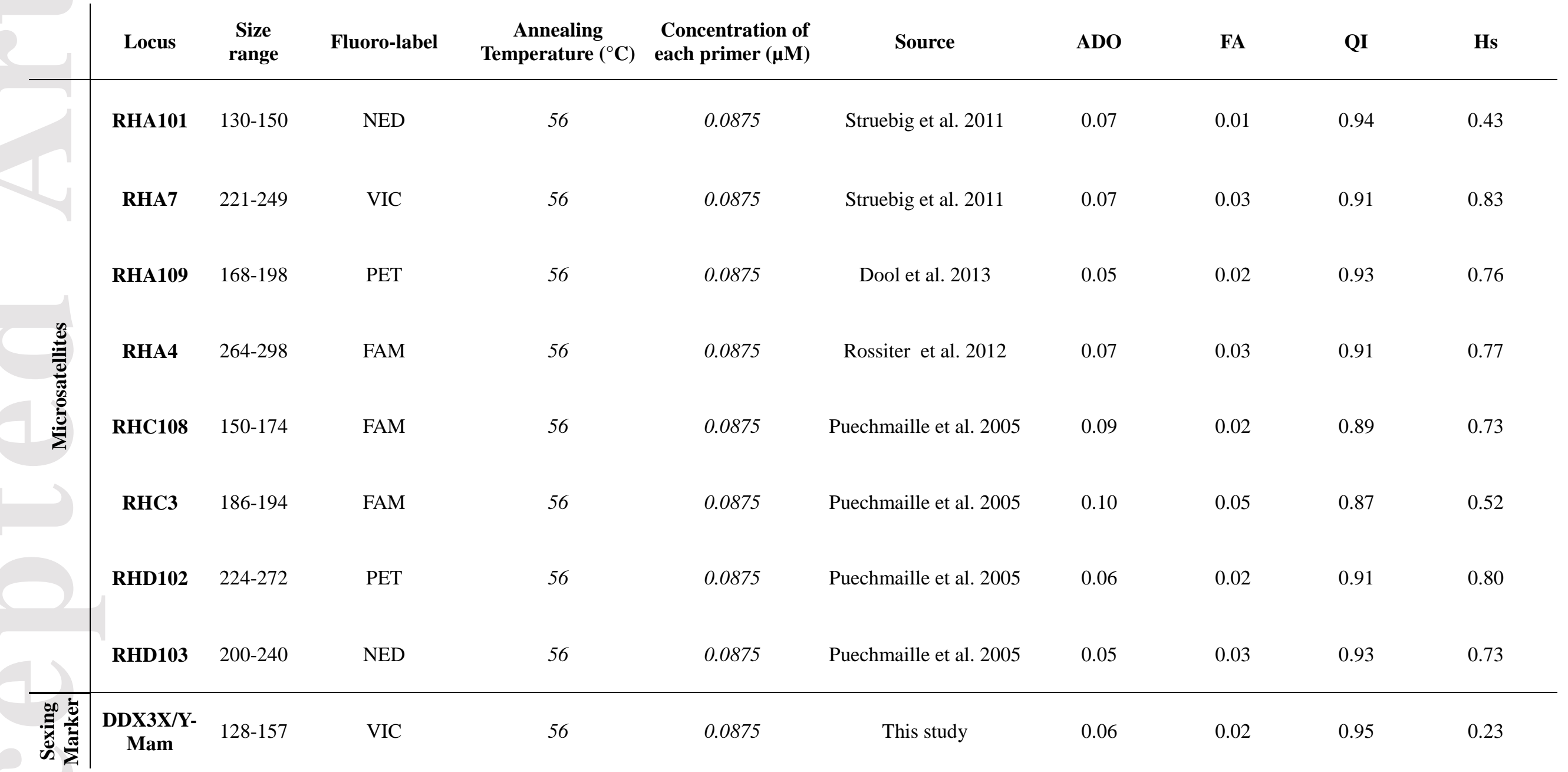


Table 2: Colonies' demographic and genetic characteristics. The number of adults visually counted, the number of unique genotypes (and corresponding proportion of males) and population sizes estimated genetically before and after parturition are presented separately. * indicates that population size estimates did not meet the homogeneous sampling assumption. Genetic characteristics were calculated using microsatellites data from

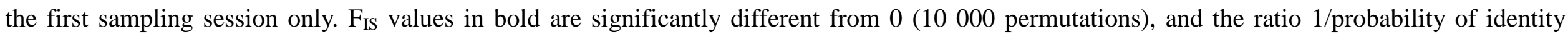
considering unrelated (PID-rand) or siblings (PID-sibs) individuals.

\begin{tabular}{|c|c|c|c|c|c|c|c|c|c|c|c|}
\hline \multirow{3}{*}{ Colony } & \multicolumn{4}{|c|}{ Before parturition } & \multicolumn{4}{|c|}{ After parturition } & \multicolumn{3}{|c|}{ Genetic characteristics } \\
\hline & \multirow{2}{*}{ Count } & \multirow{2}{*}{$\begin{array}{l}\text { Number of } \\
\text { genotypes } \\
\text { (sex-ratio) }\end{array}$} & \multicolumn{2}{|c|}{ Population size estimate $(95 \% \mathrm{CI})$} & \multirow{2}{*}{ Count } & \multirow{2}{*}{$\begin{array}{l}\text { Number of } \\
\text { genotypes } \\
\text { (sex-ratio) }\end{array}$} & \multicolumn{2}{|c|}{ Population size estimate $(95 \% \mathrm{CI})$} & \multirow{2}{*}{ FIS } & \multirow{2}{*}{ 1/PIDunb } & \multirow{2}{*}{ 1/PIDsib } \\
\hline & & & Females + males & Females only & & & Females + males & Females only & & & \\
\hline Pic1 & 5 & $8(0.0 \%)$ & $24(12-49)$ & $24(12-49)$ & 5 & $4(25.0 \%)$ & NA & NA & 0.05085 & $7.03 \times 10^{10}$ & $6.75 \times 10^{2}$ \\
\hline Pic2 & 16 & $21(9.5 \%)$ & $24 *(21-30)$ & $22 *(19-27)$ & 26 & $20(35.0 \%)$ & $32(24-60)$ & $20(15-43)$ & -0.0254 & $2.02 \times 10^{7}$ & $4.13 \times 10^{2}$ \\
\hline Pic3 & 60 & $53(20.8 \%)$ & $60 *(55-69)$ & $47 *(43-53)$ & 60 & $45(15.6 \%)$ & $54 *(48-64)$ & $44 *(39-52)$ & -0.00802 & $3.36 \times 10^{8}$ & $1.24 \times 10^{3}$ \\
\hline Pic4 & 45 & $43(20.9 \%)$ & $90(67-155)$ & $67(49-121)$ & 21 & $17(23.5 \%)$ & $21 *(18-29)$ & $15(13-21)$ & 0.05155 & $2.12 \times 10^{8}$ & $1.02 \times 10^{3}$ \\
\hline Pic5 & 40 & $34(14.7 \%)$ & $60 *(46-99)$ & $50 *(38-87)$ & 6 & $27(18.5 \%)$ & $40(32-62)$ & $30(24-45)$ & 0.06916 & $1.09 \times 10^{8}$ & $7.86 \times 10^{2}$ \\
\hline Pic6 & 70 & $50(12.0 \%)$ & $87(69-128)$ & $73(58-108)$ & 101 & $62(12.9 \%)$ & $65^{*}(62-70)$ & $56 *(54-60)$ & 0.09212 & $7.25 \times 10^{7}$ & $7.87 \times 10^{2}$ \\
\hline Pic7 & 30 & $36(36.1 \%)$ & $58(46-89)$ & $33(27-53)$ & 20 & $24(33.3 \%)$ & $28 *(25-35)$ & $18 *(16-22)$ & 0.01676 & $1.06 \times 10^{8}$ & $8.00 \times 10^{2}$ \\
\hline Pic8 & 35 & $35(17.1 \%)$ & $38 *(35-44)$ & $31 *(29-36)$ & 50 & $30(16.7 \%)$ & $40(33-55)$ & $32 *(27-45)$ & -0.069 & $1.59 \times 10^{8}$ & $8.20 \times 10^{2}$ \\
\hline Pic9 & 55 & $36(8.3 \%)$ & $54 *(44-79)$ & $49 *(40-72)$ & 25 & $33(39.4 \%)$ & $45^{*}(37-62)$ & $26(21-38)$ & -0.00381 & $2.36 \times 10^{8}$ & $1.20 \times 10^{3}$ \\
\hline Pic10 & 35 & $37(32.4 \%)$ & $44(39-55)$ & $30(26-38)$ & 40 & $28(32.1 \%)$ & $41(33-63)$ & $26(21-40)$ & -0.01185 & $8.93 \times 10^{7}$ & $8.20 \times 10^{2}$ \\
\hline Pic11 & 37 & $33(18.2 \%)$ & $44(37-59)$ & $34(29-45)$ & 7 & $17(17.6 \%)$ & $18 *(17-21)$ & $15^{*}(14-17)$ & -0.01587 & $4.93 \times 10^{7}$ & $7.59 \times 10^{2}$ \\
\hline Pic12 & 91 & $60(20.0 \%)$ & $65^{*}(61-72)$ & $53^{*}(49-59)$ & 50 & $62(16.1 \%)$ & $68 *(63-74)$ & $56 *(52-61)$ & -0.03741 & $1.27 \times 10^{8}$ & $9.58 \times 10^{2}$ \\
\hline Pic13 & 50 & $51(15.7 \%)$ & $58 *(53-66)$ & $48 *(44-55)$ & 25 & $45(13.3 \%)$ & $60(52-78)$ & $50(43-63)$ & 0.00619 & $1.79 \times 10^{7}$ & $5.95 \times 10^{2}$ \\
\hline Pic14 & 80 & $63(22.2 \%)$ & $108(88-151)$ & $86(68-129)$ & 45 & $25(20.0 \%)$ & $58(40-135)$ & $41(28-98)$ & -0.031 & $2.94 \times 10^{7}$ & $8.05 \times 10^{2}$ \\
\hline Pic15 & 5 & $10(50.0 \%)$ & $35(17-59)$ & $19(7-29)$ & 10 & $16(31.3 \%)$ & $85(36-84)$ & $60(21-59)$ & 0.01548 & $5.15 \times 10^{8}$ & $4.52 \times 10^{2}$ \\
\hline Pic16 & 222 & $200(39.0 \%)$ & $235 *(222-253)$ & $140 *(131-152)$ & 215 & $143(32.2 \%)$ & $152 *(146-159)$ & $101 *(97-105)$ & -0.00448 & $7.90 \times 10^{7}$ & $1.09 \times 10^{3}$ \\
\hline Pic17 & 41 & $39(25.6 \%)$ & $51^{*}(44-67)$ & $41^{*}(33-59)$ & 3 & $24(16.7 \%)$ & $36^{*}(28-57)$ & $29 *(23-48)$ & -0.01043 & $1.46 \times 10^{6}$ & $2.77 \times 10^{2}$ \\
\hline Pic18 & 40 & $55(16.4 \%)$ & $67(60-80)$ & $54(49-64)$ & 60 & $33(3.0 \%)$ & $45^{*}(37-62)$ & $43 *(36-59)$ & 0.04052 & $9.37 \times 10^{7}$ & $9.94 \times 10^{2}$ \\
\hline Pic 19 & 10 & $12(41.7 \%)$ & $17(13-34)$ & $9(7-20)$ & 10 & $4(25.0 \%)$ & $8(5-29)$ & $6(3-23)$ & 0.12659 & $1.09 \times 10^{8}$ & $3.04 \times 10^{2}$ \\
\hline
\end{tabular}



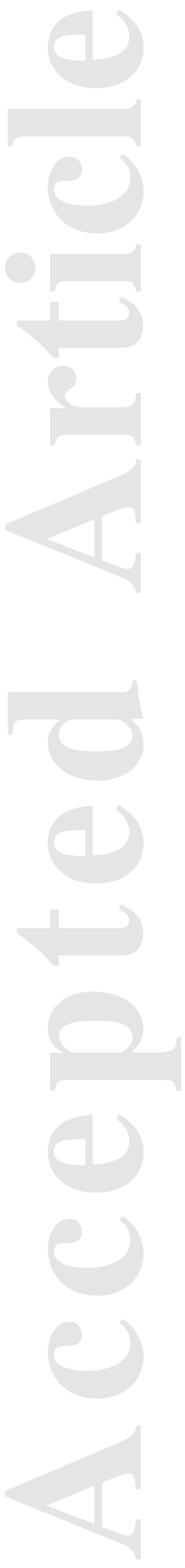
Table 3: Number of adults sampled only before parturition $v s$ in both sessions, depending on their sex and, for females, their assignment as a mother. *: significant Chi square-test (pvalues $<0.001$ )

\begin{tabular}{|c|c|c|c|}
\hline & $\begin{array}{c}\text { Sampled only } \\
\text { before parturition }\end{array}$ & $\begin{array}{l}\text { Sampled before and } \\
\text { after parturition }\end{array}$ & \\
\hline Males & 170 & 44 & $\Longrightarrow$ \\
\hline Unassigned females & 321 & 167 & $\bar{u}^{*}$ \\
\hline Mothers & 63 & 111 & ${ }^{*}$ \\
\hline
\end{tabular}


Figure 1: Location of the 19 monitored colonies (Picardie, France)

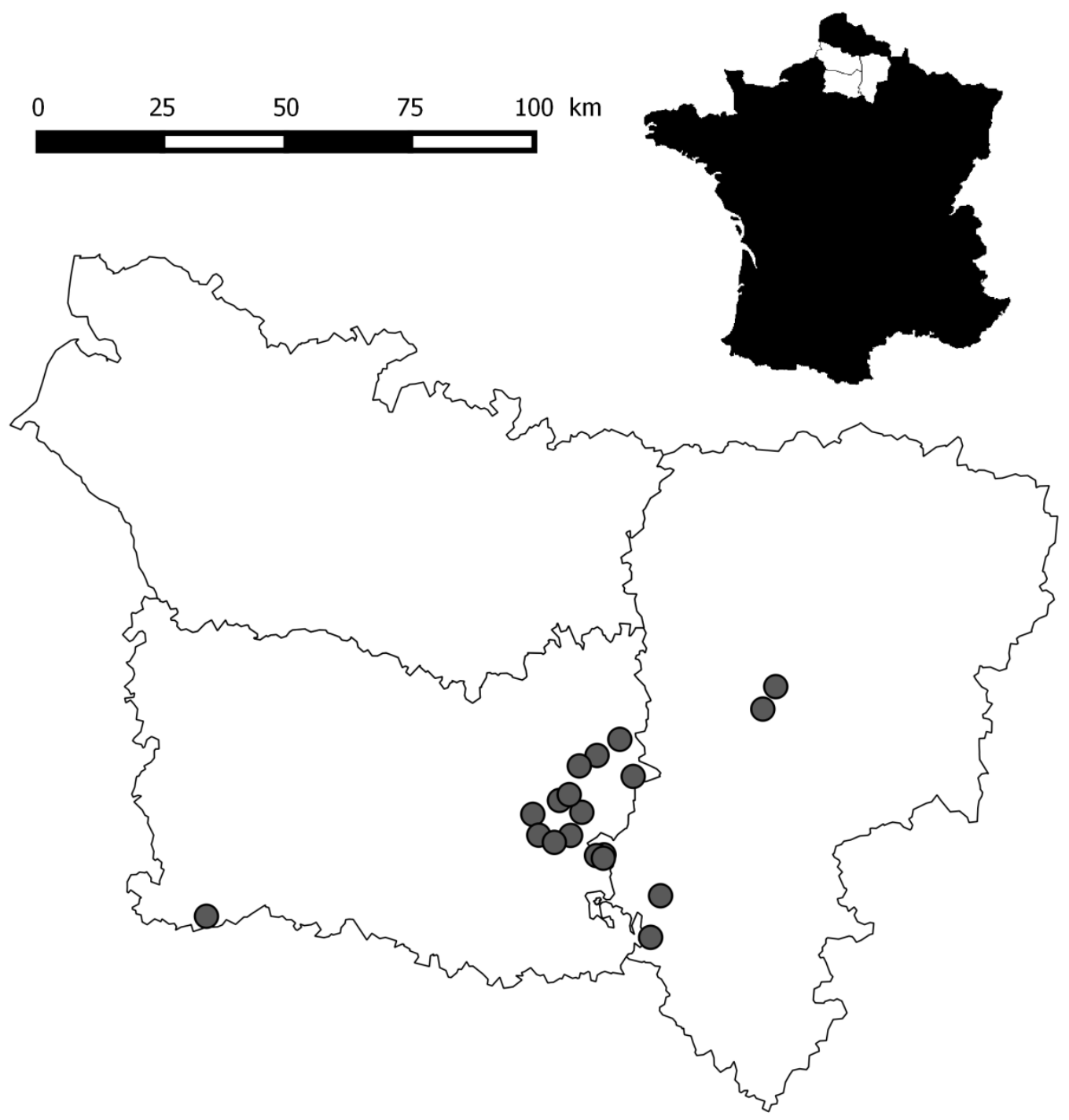


Figure 2: Colony sizes estimated by visual counts or CMR approach for both sampling sessions. CMR models were applied to all sampled individuals and to adult females only. Error bars represent standard error. Letters represent significant differences (Least-square means, p-values $<0.05$ )

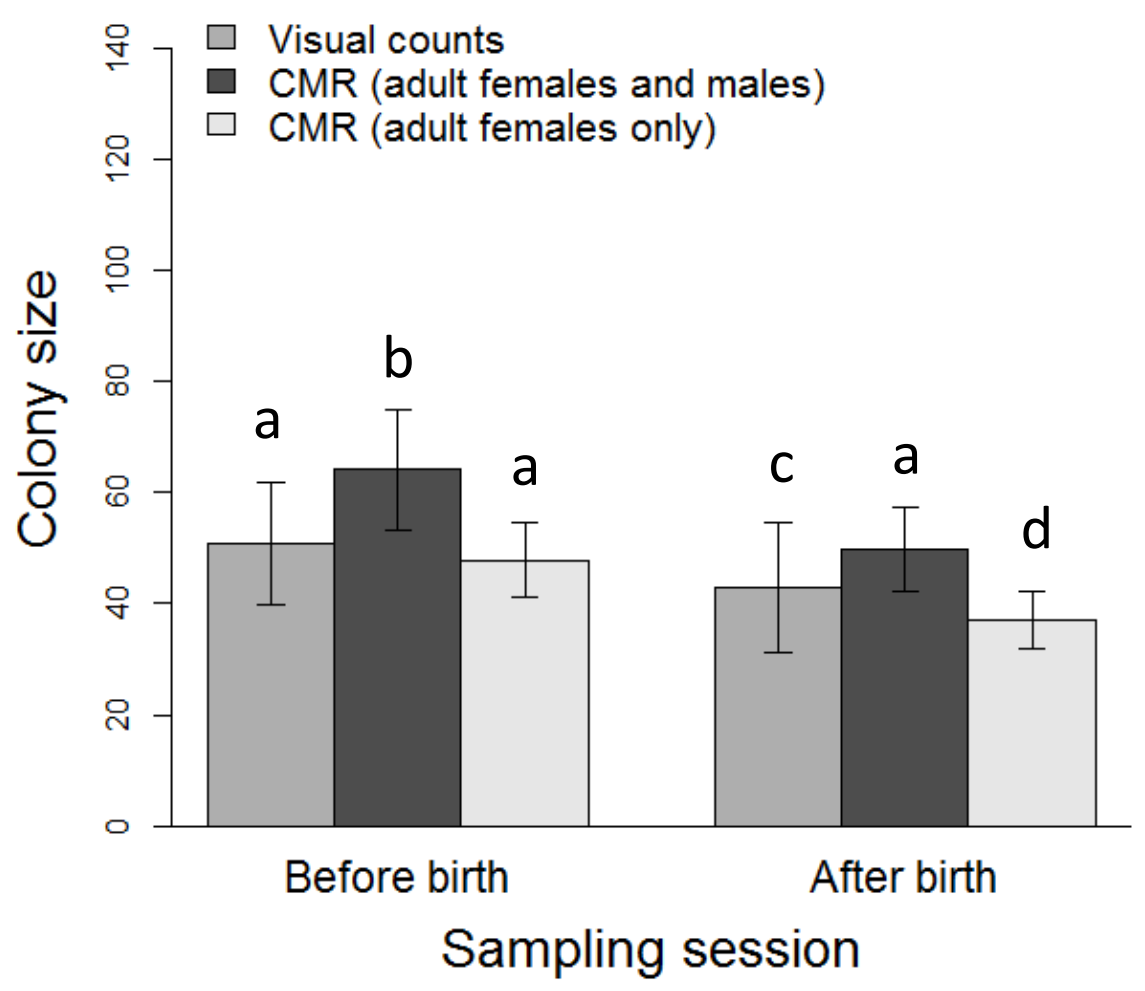


Figure 3: Number of captures for males, non-mother females, and females assigned as mothers for both sampling sessions. Error bars correspond to the standard error. Different letters correspond to a significant difference (Least-square means, $p$-values < 0.05 )

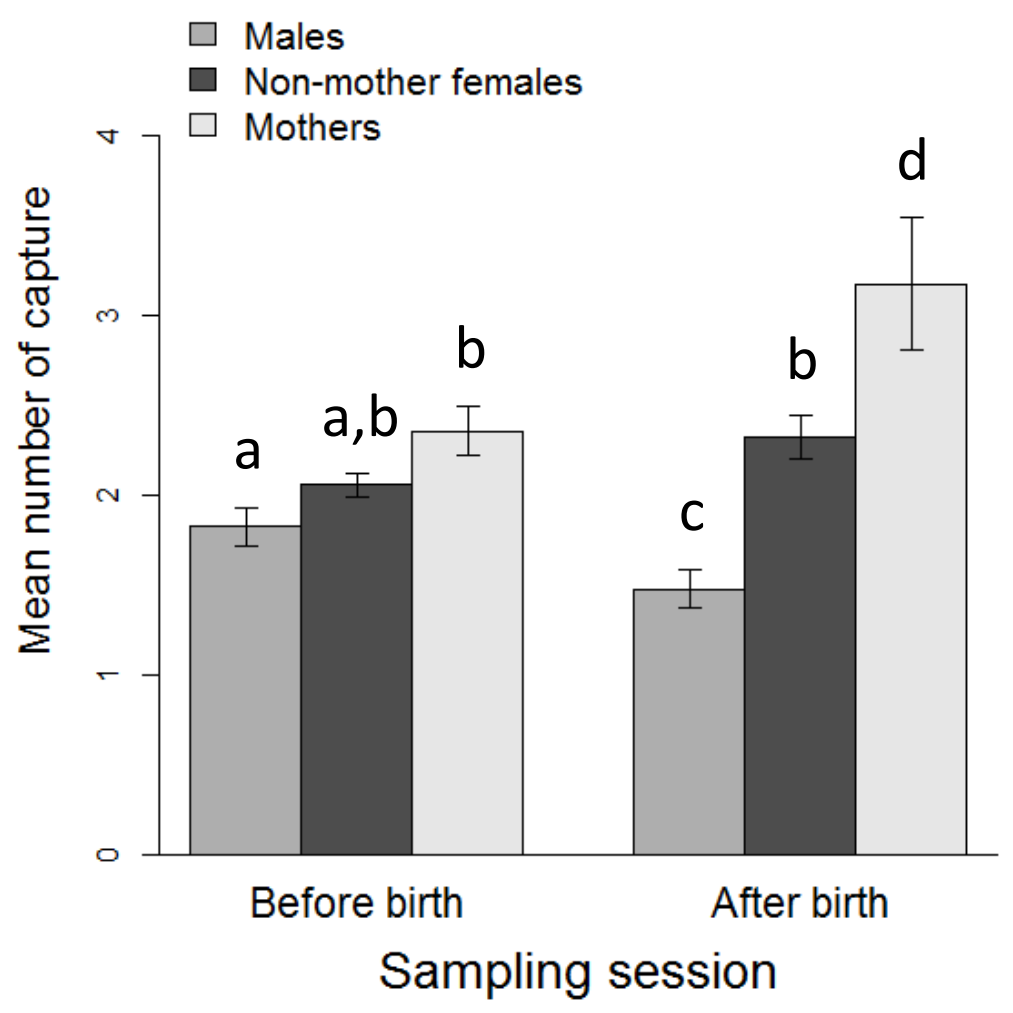

